\title{
Combination Antifungal Therapy for Invasive Aspergillosis Revisited
}

\author{
Anil A. Panackal, M.D. ${ }^{1,2}$ \\ ${ }^{1}$ Laboratory of Clinical Infectious Diseases, National Institute of Allergy and Infectious Diseases, \\ National Institutes of Health, Bethesda, MD, USA \\ 2Division of Infectious Diseases, Department of Medicine, F. Hèbert School of Medicine, \\ Uniformed Services University of the Health Sciences (USUHS)
}

\begin{abstract}
Invasive aspergillosis (IA) causes significant morbidity and mortality among immunocompromised hosts. Combination therapy with mold-active triazoles and echinocandins has been used with the hope of improving outcomes over monotherapy, especially in the setting of refractory disease. Herein, I update our prior systematic review and meta-analysis on combination therapy for salvage IA in the context of the recently published randomized clinical trial of combination therapy for primary IA. Clinicians should consider combination antifungals for IA in refractory disease despite immune reconstitution when there are concerns for resistance or pharmacokinetic variability.
\end{abstract}

The controversies surrounding the use of combination antifungal therapy for invasive aspergillosis (IA) are ongoing. Initially sparked by encouraging in vitro and animal studies that demonstrated synergistic or additive effects when combining a mold-active triazole (itraconazole, voriconazole, or posaconazole) or an amphotericin B with an echinocandin (caspofungin, micafungin, or anidulafungin) [1-3], such as the neutropenia rabbit model of IA in which a correlation between in vitro synergy in plasma and fungal tissue burden decrease was noted [4], application to human clinical care in patients with IA became part of the approach to management in several small studies. Nonetheless, despite a recently published large, randomized clinical trial (RCT) by Marr et al comparing voriconazole and anidulafungin to voriconazole alone, which targeted primary IA cases, and a systematic review and meta-analysis by Panackal et al comparing mold-active triazoles or lipid amphotericin B plus an echinocandin to non-echinocandin-based monotherapy for acute IA, targeting mostly salvage IA (75\% of studies for refractory IA), clinicians are still left in a quandary $[5,6]$.

The RCT by Marr et al did not find overall significance as a primary outcome but did find significance favoring combination therapy with voriconazole and andidulafungin over voriconazole for primary IA during a post hoc subgroup analysis among galactomannan positive patients only. Six-week all-cause mortality was $15.7 \%$ (17 of 108) in the

Corresponding Author: Anil A. Panackal, M.D., Staff Clinician, NIAID/NIH, Clinical Center, 10 Center Drive, Bethesda, MD 20814, anil.panackal@nih.gov. 
combination group compared with $27.3 \%$ (30 of 110) in the monotherapy group (difference, $-11.6 \%$ [CI, -22.7 to -0.4$] ; p=0.037$ ). The author concluded that the RCT was underpowered due to the lower than expected treatment effect difference observed in this primary endpoint. The 6-week endpoint was selected based upon the recent movement of the mycological community away from subjective composite endpoints and towards objective measures that are most likely to encompass attributable IA mortality with a lessened concern for competing risks [7]. In fact, most deaths at 6 weeks (84.6-88.5\%) were found by the data review committee to be attributable to IA. Nonetheless, overall mortality rates at 6 weeks were $19.3 \%$ (26 of 135) for combination therapy and 27.5\% (39 of 142) for monotherapy (difference, -8.2 percentage points [95\% CI, -19.0 to 1.5$]$; $p=0.087$ ). Notably, the duration of therapy was shorter in the combination arm (median=14 [1-29] days) compared to the monotherapy arm (median=42 [1-48] days) and the incidence of drug related adverse events due to voriconazole that necessitated reducing the antifungal dosage and consequent levels was $20 \%$, which may have affected the results. Indeed, Park et al found that therapeutic drug level monitoring (TDM) of voriconazole resulted in significantly fewer drug discontinuation due to adverse events ( $4 \%$ vs. $17 \%, p=0.02)$ and significantly improved complete/partial treatment response at 3 months in invasive fungal infections ( $81 \%$ vs. $57 \%, p=0.04$ ) [8]. Nevertheless, this RCT did try to maximize efficacy by selecting a homogeneous population with lower risk for death due to underlying conditions $[5,9]$.

The heterogeneity noted in the systematic review and meta-analysis by Panackal et al was challenging. Attempts at reducing heterogeneity by using propensity score matching based upon variables such as neutropenia would have reduced the number of studies and thereby power significantly. Moreover, we attempted to obtain individualized patient data on this topic from a variety of surveillance efforts but were unable to do so. In addition, 12-week mortality and composite scores were the most consistent endpoints across the majority of studies, as these preceded the study by Wingard et al that noted that the etiology for IA death was most attributed to IA if death occurred by 6 weeks ( $89 \%$ of cases), derived from the Herbrecht et al pivotal voriconazole versus amphotericin B for IA trial that led to voriconazole's approval for primary IA [7, 10]. In this study, we found that there was an $80 \%$ increase in 12-week survival $(p=0.02)$ when restricting to high quality studies that primarily included combination for refractory IA rather than for drug intolerance. The subjective endpoint of 12-week global success was marginal ( $\mathrm{OR}=1.72$, 95\%CI 0.96-3.09, $p=0.07)$ likely due to drop outs decreasing evaluability. We clearly have asserted that these results must be interpreted with caution, given the heterogeneity inherent with meta-analyses, particularly among diverse patient groups whose management and case definitions have changed over time (i.e., a cohort effect) $[6,11,12]$. Furthermore, TDM was not consistently done across studies for voriconazole with variable levels due partly to non-linear pharmacokinetics, though it has been reported that TDM may improve the therapeutic index of voriconazole [8]. In addition, voriconazole may have a variety of drug interactions with other drugs that affect the cytochrome p450 hepatic system (e.g., carbamazepine) and there may be population heterogeneity in related polymorphisms (e.g., CYP 2C19), which drive its levels down [13]. Although, other antifungal ex vivo PK/PD assays are not routinely done, tissue penetration of the lung - the most common site of IA - is comparable amongst the antifungal classes: $0.3-3.2 \mathrm{X}$ for voriconazole, up to $3 \mathrm{X}$ depending on the lipid 
formulation of amphotericin B, and 1.1-6.2X for echinocandins that of simultaneous plasma concentrations $(\mu \mathrm{g} / \mathrm{ml})[14]$. Moreover, each antifungal class may have variable immunomodulatory effects, as has been previously reviewed [15]. Finally, the benefits of the combination antifungal approach have been re-enforced in the setting of the emergence of azole resistant Aspergillus by expert opinion, but implementation must be weighed against potential costs and toxicity [16-19].

Nevertheless, a consistent evidence basis for the use of combination antifungals for IA has not been uniformly found. For example, recently there have been a few additional studies on the topic published [18, 20-23]. First, Wattier et al found that combination antifungal therapy for IA in the pediatric hematology population was associated increased risk for adverse events $(\mathrm{RR}=1.98 ; 95 \% \mathrm{CI}, 1.06-3.68 ; p=0.031)$ without a discernable difference in the 12week survival outcome [20]. Similarly, in this same host population, Domenech et al reported combination therapy may have had a role in improving the outcome in $58 \%$ of their patients, but this was a small historical study and so it is difficult to draw conclusions [21]. Cornely et al evaluated micafungin monotherapy for salvage IA therapy, but among the 301 screened patients, only 17 were not receiving combination antifungal therapy so only these could be included in this study; three patients in the micafungin treatment arm $(25.0 \%$; $95 \%$ CI: 5.5-57.2) and three patients in the control arm (60.0\%; 95\% CI: 14.7-94.7) had successful therapy at end of therapy (EOT) but the low numbers preclude definitive conclusions. Unfortunately, the results on the majority receiving combination therapy for salvage IA are not listed in this publication [22]. Finally, Raad et al evaluated 181 patients with haematological malignancies with IA who received primary or salvage therapy with caspofungin, voriconazole or a combination of both for at least 7 days. For primary therapy, although all 3 interventions showed no significant differences in response at the EOT (caspofungin, voriconazole and combination therapy were associated with response rates of $27 \%, 47 \%$ and $55 \%$, respectively ( $p=0.2$ ), those receiving voriconazole had $80 \%$ lower IAassociated mortality than those receiving caspofungin $(8 \%$ vs. $47 \% ; P=0.003)$ or combination therapy ( $8 \%$ vs. $27 \% ; P=0.03$ ) in their multivariate competing risk model. For salvage therapy, caspofungin, voriconazole and combination therapy were also associated with non-differential complete/partial responses at EOT of $27 \%, 47 \%$ and $55 \%$, respectively $(p=0.2)$. Unfortunately, no significant mortality rates among these groups were found ( $p=$ 0.32 ), but the adverse event rates were $6 \%$ for caspofungin, $17 \%$ for voriconazole and $37 \%$ for the combination with the difference between caspofungin and the combination being statistically significant $(P=0.02)$. Interestingly, ICU admission was independently associated with IA-associated mortality in bother the primary and salvage setting. Of course, all of these studies were retrospective observational and therefore prone to selection bias by nature.

In summary, "the jury is still out" on whether combination therapy is the way to go with IA. However, the RCT by Marr et al and the systematic review and meta-analysis by Panackal et al provide insights into management. First, combination therapy may benefit a subset of patients that are serum galatomannan positive while use in refractory IA may improve outcomes, particularly once there is neutrophil recovery and the patient is weaned off immunosuppression when there are concerns for antifungal resistance or poor drug levels. Specifically, combination therapy for IA probably has a role when there is suspected drug 
resistance (e.g., Cyp51a mutations) [3] and broad initial coverage followed by rapid deescalation pending pathogen identification for salvage therapy in the setting of refractory disease or for drug intolerance in the setting of adverse effects. Indeed, as I alluded to in our earlier study, the probability of achieving another appropriately powered and comparative, double-blinded, multicenter trial for primary or, for that matter, refractory IA therapy is infinitesimally small, given practical and logistical concerns. Nevertheless, our studies need to be designed by carefully choosing meaningful homogeneous and objective endpoints (e.g., 6-week mortality) in order to provide direct and reproducible comparisons in the future. Finally, the decreased observed toxicity with the echinocandins make this drug class appealing especially if combination therapy is pursued.

\section{Acknowledgements}

This research was supported by extramural grants AI001123-01 and AI001124-01 from the National Institute of Allergy and Infectious Diseases (NIAID) at the National Institutes of Health (NIH), and, in part, by the Intramural Research Program of the NIAID. In addition, the views herein do not reflect the official opinions of the Uniformed Services University (USUHS) or the Department of Defense.

\section{References}

1. Kirkpatrick WR, Perea S, Coco BJ, Patterson TF. Efficacy of caspofungin alone and in combination with voriconazole in a Guinea pig model of invasive aspergillosis. Antimicrobial agents and chemotherapy. 2002; 46:2564-8. [PubMed: 12121933]

2. Nagasaki Y, Eriguchi Y, Uchida Y, et al. Combination therapy with micafungin and amphotericin B for invasive pulmonary aspergillosis in an immunocompromised mouse model. The Journal of antimicrobial chemotherapy. 2009; 64:379-82. [PubMed: 19465436]

3. Seyedmousavi S, Meletiadis J, Melchers WJ, Rijs AJ, Mouton JW, Verweij PE. In vitro interaction of voriconazole and anidulafungin against triazole-resistant Aspergillus fumigatus. Antimicrobial agents and chemotherapy. 2013; 57:796-803. [PubMed: 23183435]

4. Petraitis V, Petraitiene R, Sarafandi AA, et al. Combination therapy in treatment of experimental pulmonary aspergillosis: synergistic interaction between an antifungal triazole and an echinocandin. The Journal of infectious diseases. 2003; 187:1834-43. [PubMed: 12792859]

5. Marr KA, Schlamm HT, Herbrecht R, et al. Combination antifungal therapy for invasive aspergillosis: a randomized trial. Ann Intern Med. 2015; 162:81-9. [PubMed: 25599346]

6. Panackal AA, Parisini E, Proschan M. Salvage combination antifungal therapy for acute invasive aspergillosis may improve outcomes: a systematic review and meta-analysis. Int J Infect Dis. 2014; 28:80-94. [PubMed: 25240416]

7. Wingard JR, Ribaud P, Schlamm HT, Herbrecht R. Changes in causes of death over time after treatment for invasive aspergillosis. Cancer. 2008; 112:2309-12. [PubMed: 18338758]

8. Park WB, Kim NH, Kim KH, et al. The effect of therapeutic drug monitoring on safety and efficacy of voriconazole in invasive fungal infections: a randomized controlled trial. Clinical infectious diseases : an official publication of the Infectious Diseases Society of America. 2012; 55:1080-7. [PubMed: 22761409]

9. Marr KA, Schlamm H, Rottinghaus ST, et al. A randomised, double-blind study of combination antifungal therapy with voriconazole and anidulafungin versus voriconazole monotherapy for primary treatment of invasive aspergillosis. ECCMID. (London, UK).

10. Herbrecht R, Denning DW, Patterson TF, et al. Voriconazole versus amphotericin B for primary therapy of invasive aspergillosis. N Engl J Med. 2002; 347:408-15. [PubMed: 12167683]

11. Ascioglu S, Rex JH, de Pauw B, et al. Defining opportunistic invasive fungal infections in immunocompromised patients with cancer and hematopoietic stem cell transplants: an international consensus. Clinical infectious diseases : an official publication of the Infectious Diseases Society of America. 2002; 34:7-14. [PubMed: 11731939] 
12. De Pauw B, Walsh TJ, Donnelly JP, et al. Revised definitions of invasive fungal disease from the European Organization for Research and Treatment of Cancer/Invasive Fungal Infections Cooperative Group and the National Institute of Allergy and Infectious Diseases Mycoses Study Group (EORTC/MSG) Consensus Group. Clinical infectious diseases : an official publication of the Infectious Diseases Society of America. 2008; 46:1813-21. [PubMed: 18462102]

13. Theuretzbacher U, Ihle F, Derendorf H. Pharmacokinetic/pharmacodynamic profile of voriconazole. Clinical pharmacokinetics. 2006; 45:649-63. [PubMed: 16802848]

14. Felton T, Troke PF, Hope WW. Tissue penetration of antifungal agents. Clinical microbiology reviews. 2014; 27:68-88. [PubMed: 24396137]

15. Ben-Ami R, Lewis RE, Kontoyiannis DP. Immunocompromised hosts: immunopharmacology of modern antifungals. Clinical infectious diseases : an official publication of the Infectious Diseases Society of America. 2008; 47:226-35. [PubMed: 18540822]

16. Verweij PE, Ananda-Rajah M, Andes D, et al. International expert opinion on the management of infection caused by azole-resistant Aspergillus fumigatus. Drug Resist Updat. 2015; 21-22:30-40. [PubMed: 26282594]

17. van der Linden JW, Arendrup MC, Warris A, et al. Prospective multicenter international surveillance of azole resistance in Aspergillus fumigatus. Emerg Infect Dis. 2015; 21:1041-4. [PubMed: 25988348]

18. Candoni A, Aversa F, Busca A, et al. Combination antifungal therapy for invasive mould diseases in haematologic patients. An update on clinical data. J Chemother. 2015; 27:1-12. [PubMed: 25466728]

19. Martin-Pena A, Aguilar-Guisado M, Espigado I, Cisneros JM. Antifungal combination therapy for invasive aspergillosis. Clinical infectious diseases : an official publication of the Infectious Diseases Society of America. 2014; 59:1437-45. [PubMed: 25048847]

20. Wattier RL, Dvorak CC, Hoffman JA, et al. A Prospective, International Cohort Study of Invasive Mold Infections in Children. J Pediatric Infect Dis Soc. 2015; 4:313-22. [PubMed: 26582870]

21. Domenech C, Leick-Courtois C, Bienvenu AL, et al. Improvement in the Outcome of Invasive Aspergillosis in a Pediatric Hematology Department: A 10-Year Review. J Pediatr Hematol Oncol. 2015; 37:560-5. [PubMed: 26165404]

22. Cornely OA, Meems L, Herbrecht R, Viscoli C, van Amsterdam RG, Ruhnke M. Randomised, multicentre trial of micafungin vs. an institutional standard regimen for salvage treatment of invasive aspergillosis. Mycoses. 2015; 58:58-64. [PubMed: 25530265]

23. Raad, Zakhem AE, Helou GE, Jiang Y, Kontoyiannis DP, Hachem R. Clinical experience of the use of voriconazole, caspofungin or the combination in primary and salvage therapy of invasive aspergillosis in haematological malignancies. Int J Antimicrob Agents. 2015; 45:283-8. [PubMed: 25455847] 